\title{
TERAPIA EMDR EN EL TRASTORNO LÍMITE DE PERSONALIDAD EMDR THERAPY IN BORDERLINE PERSONALITY DISORDER
}

\author{
Dolores Mosquera a ${ }^{\text {Y }}$ AnABel GonzÁlez a \\ a Instituto para el tratamiento del trauma y los trastornos de personalidad (INTRA-TP). \\ doloresmosquera@gmail.com
}

Cómo referenciar este artículo/How to reference this article:

Mosquera, D. y González, A. (2013). Terapia EMDR en el trastorno límite de personalidad [EMDR therapy in borderline personality disorder]. Acción Psicológica, 10(1), 85-96. http://dx.doi.org/10.5944/ ap.10.1.7036

\section{Resumen}

Los trastornos de personalidad se han asociado a problemas de apego y trauma temprano, siendo de todos ellos el trastorno límite el que presenta unos porcentajes más elevados. La aplicación de terapias orientadas al trauma como EMDR (Desensibilización y Reprocesamiento por Movimientos Oculares) en estos trastornos ofrece interesantes posibilidades para su abordaje.

Palabras clave: EMDR; trastorno de personalidad; trastorno límite de personalidad; Experiencias traumáticas.

\begin{abstract}
Personality disorders have been related with early attachment problems and trauma being. borderline personality disorder the one that presents the higher percentage of these factors. EMDR therapy is a recently developed psychotherapeutic approach, with increasing recognition for the treatment of traumatic experiences that offers interesting possibilities for it's application in personality disorders.
\end{abstract}

Keywords: EMDR; Personality Disorder; Borderline Personality Disorder; Traumatic Experiences.

\section{Introducción}

La terapia EMDR ha obtenido reconocimiento internacional como tratamiento de elección para el trastorno de estrés postraumático, aunque su utilización se ha extendido a numerosas patologías. En este artículo se explicará cómo se entiende el trastorno límite de personalidad desde esta perspectiva.

Las siglas EMDR corresponden a desensibilización y reprocesamiento por movimiento ocular. El modelo teórico se basa en el sistema de procesamiento de información intrínseco del cerebro y cómo se almacenan las memorias. Los síntomas y problemas actuales del paciente se consideran resultado de experiencias perturbadoras previas que no fueron procesadas adecuadamente, y permanecen en un estado específico, es decir, están almacenadas disfuncionalmente (Shapiro, 2001, 2007)

La esencia del EMDR es la transmutación de esta información almacenada disfuncionalmente a un modo de almacenamiento adaptativo que promueva la salud psicológica. En condiciones naturales, el sistema innato de procesamiento de información (AIP: Adaptive Information Processing System) asimila las experiencias recientes en redes neurales ya exis- 
tentes. Estas redes neurales son la base de la percepción, de actitudes y conductas. Cuando este sistema funciona de modo adecuado, las experiencias nuevas son asimiladas, integrándose y conectándose con información que esta almacenada en estas redes neurales. Esta conexión con información previa nos permite dar sentido a la experiencia, nos hace aprender y ayuda a guiarnos en el futuro (Shapiro, 2001).

Pero ante un evento traumático este sistema de procesamiento adaptativo de información (PAI) se bloquea, y la información, en lugar de integrarse con el resto de las redes de memoria, se almacena de modo disfuncional.

La memoria traumática tiene características diferentes a la memoria ordinaria. Mientras que la memoria ordinaria tiene menos viveza perceptiva y está conectada con el resto de la experiencia, la memoria traumática es incompleta o incoherente; parcializada hacia los detalles que son significativos emocionalmente, amenazantes, nuevos o de especial importancia para el individuo. La diferencia entre la memoria traumática y la ordinaria es la que existe entre revivir el suceso traumático (igual que allí y entonces) y recordar que es lo que nos sucedió, pero que ya es pasado.

Para el abordaje de cualquier patología lo fundamental es seleccionar las dianas adecuadas, aquellos recuerdos clave que son la base del problema actual, así como eventos posteriores que están también asociados en este mismo cluster o racimo de sucesos conectados, y que funcionan como memorias alimentadoras.

\section{Aplicaciones clínicas de la terapia EMDR}

EMDR es una terapia orientada al tratamiento del trauma, aunque su aplicación se ha ido extendiendo a muy distintas patologías. En los últimos años su eficacia ha sido avalada por un gran número de estudios empíricos y hoy en día está reconocida como una terapia de elección para el tratamiento del trastorno de estrés postraumático. En diversas Guías Clínicas se ha reconocido como una terapia efectiva y se considera un tratamiento de Categoría A (fuer- temente recomendados) para el tratamiento del trauma (Foa, Keane y Friedman, 2000)

Varios metaanálisis revisan los estudios disponibles concluyendo que EMDR es de eficacia igual o superior a terapias de orientación cognitivo-conductual (Bisson y Andrew, 2007; Bradley, Greene, Russ, Dutra, y Westen, 2005; Davidson y Parker, 2001; Seidler y Wagner, 2006), sobre todo cuando es aplicado adecuadamente (Maxfield y Hyer, 2002). En algunos estudios se apuntan varias de las ventajas de EMDR: una mayor eficiencia y rapidez (Van Etten y Taylor, 1998).

\section{Como funciona EMDR}

La Terapia de Desensibilización y Reprocesamiento por Movimientos oculares (EMDR; Shapiro, 2001) se basa en el Modelo de Procesamiento de Información a un Estado Adaptativo (PAI). Según este modelo, en situaciones traumáticas la información queda almacenada disfuncionalmente. El objetivo del tratamiento es acceder a estas memorias almacenadas de modo disfuncional, y procesarlas, con lo que las sensaciones, emociones y elementos cognitivos asociados a estas memorias se transforman a un estado adaptativo.

Un ingrediente activo de esta terapia es, como comentábamos, el uso de movimientos oculares, sonidos bilaterales o estimulación táctil alternantes (lo que denominamos estimulación bilateral). La estimulación bilateral activa y facilita la conexión entre la información almacenada disfuncionalmente y el resto de redes neurales. Los recuerdos traumáticos se integran así con información adaptativa (como por ejemplo otras experiencias en las que superamos situaciones similares). Los estudios neurobiológicos señalan que esto se debe a un cambio de información sensorial implícita a memorias consolidadas explícitas (Siegel, 2002; van der Kolk, 2002) y de sistemas de memoria episódica, mediada por el hipocampo, a sistemas de memoria semántica, mediados por el neocortex (Stickgold, 2002).

El concepto de trauma descrito en los criterios del Trastorno de Estrés Postraumático, es 
el que en EMDR se denomina trauma «T». Pero este concepto de trauma desde esta perspectiva se hace también extensivo a todas aquellas situaciones más relacionadas con el apego, la falta de atención o negligencia y a situaciones cotidianas que pueden jugar un papel destacable en la patología, y que se denominan traumas con $\mathrm{t}$ minúscula «t».

\section{Trauma «T» $\mathbf{T}$ TLP}

Distintos estudios han descrito una comorbilidad frecuente entre TEPT y TLP (Driessen et al, 2002; Harned, Rizvi y Linehan 2010; McLean y Gallop, 2003). Otros encuentran una relación entre TLP y abuso emocional (Kingdon et al, 2010) y distintos tipos de abuso (Grover et al., 2007; Tyrka, Wyche, Kelly, Price y Carpenter, 2009). Una historia de trauma en la infancia predice un mal pronóstico en pacientes borderline. Los síntomas de TEPT predicen junto con la sintomatología disociativa las conductas autodestructivas (Sansone, Sansone y Wiederman, 1995; Spitzer, Effler y Freyberger, 2000).

Zanarini (2000) describe una estimación de abuso sexual en la infancia en el TLP entre un 40 y un $70 \%$. Diversos estudios muestran también una relación significativa entre abuso sexual, maltrato en la infancia y TLP (Battle et al., 2004; Goodman y Yehuda, 2002; Laporte y Guttman, 1996; Rogosch y Chiccetti, 2005; Sabo, 1997; Yen et al., 2002) Algunos autores hablan de un efecto más moderado, como Fossati, Madeddu y Maffei (1999) en un metaanálisis de 21 estudios que examinaron la relación entre TLP y abuso sexual en la infancia.

Aunque la prevalencia de trauma en la infancia es alta en todos los trastornos de personalidad (Johnson, Cohen, Brown, Smailes y Bernstein, 1999), la relación parece más fuerte con el TLP que en otros trastornos de personalidad. La severidad del abuso sexual también se ha relacionado con la severidad de los rasgos límites (Silk, Lee, Hill y Lohr, 1995) y las conductas autodestructivas (Sansone, Sansone y Wiederman, 1995). Zweig-Frank y Paris (1991 encontraron que el grado de severidad del abuso podía distinguir entre individuos con TLP de aquellos sin TLP. Yen et al. (2002) destacaron que entre distintos trastornos de personalidad, los participantes con TLP refirieron la tasa más alta de exposición traumática (particularmente trauma sexual, incluyendo abuso sexual en la infancia), las tasas más altas de trastorno de estrés postraumático, y una edad más temprana del primer evento traumático.

Ball y Links (2009) revisaron la literatura sobre trauma y TLP en el contexto de los criterios clásicos de Hill (1965) para demostrar causalidad (fuerza, consistencia, especificidad, temporalidad, gradiente biológico, plausibilidad, coherencia, evidencia experimental y analogía). Estos autores demostraron que el trauma puede ser considerado un factor causal en el desarrollo del TLP, como parte de un modelo etiológico multifactorial.

\section{TLP y trauma «t»}

En EMDR se da mucha relevancia, como comentábamos, no sólo a los eventos traumáticos definidos en el TEPT («T») sino a aquellas experiencias adversas más "cotidianas». Muchas de estas experiencias están incluidas en lo que conocemos como apego disfuncional. Estos problemas de apego temprano y experiencias adversas de crianza han sido descritos por muchos autores como factores clave en el desarrollo del TLP (Bateman y Fonagy, 2004).

Sabo (1997) revisa la literatura sobre experiencias infantiles entre pacientes con TLP, y concluye que la negligencia biparental y la ausencia de figuras de apego sustitutivas son factores traumatizantes muy poderosos en el desarrollo del TLP. Otros autores han sugerido la importancia del fallo biparental en el desarrollo del Trastorno Límite (Zanarini et al., 2000; Zweig-Frank y Paris, 1991) o del tipo de vinculación parental (Guttman y Laporte 2002). Zweig-Frank y Paris (1991) hablan de escaso cuidado y sobreprotección (control sin afecto). Gunderson $(1984,1996)$ consideraba que la incapacidad de los borderline para invocar una «introyección calmante» era una consecuencia del fallo de las figuras de apego tempranas. Muchos autores han relacionado el TLP con diversos tipos de apego inseguro. 


\section{Relación entre entornos adversos en la infancia y TLP}

En el caso de los trastornos límite de la personalidad, puede resultar difícil establecer el hilo conector entre los síntomas del paciente (incluyendo las frecuentes dificultades que presentan en la relación terapéutica) y los entornos tempranos en los que crecieron, caracterizados por un alto índice de eventos traumáticos graves, pero sobre todo en la acumulación crónica y devastadora de múltiples traumas «t». La aparatosidad de los síntomas más evidentes del TLP hace que muchos profesionales se sigan centrando en el síntoma y no lleguen a entender la conexión entre éste y los estudios que hablan de una alta prevalencia de apego disfuncional y trauma temprano. Puede consultarse una revisión en profundidad de la interacción entre trauma, apego y factores biológicos en Mosquera, González y Van der Hart (2011) donde se explica además la estrecha interconexión entre la disociación y el trastorno límite.

A continuación trataremos de entender posibles experiencias tempranas desde las que se podrían desarrollar problemas que encajan en cada uno de los criterios DSM. Es importante tener en mente que cada criterio o síntoma es la manifestación de un conjunto de experiencias traumáticas que serán procesadas con EMDR.

Los criterios DSM desde el modelo del sistema de procesamiento adaptativo de información.

Los criterios diagnósticos de las clasificaciones internacionales son una guía para identificar un perfil de problemas, pero trasladar esta sintomatología a la elaboración de un plan terapéutico para trabajar con EMDR no es algo que pueda hacerse de modo automático. Revisaremos los distintos criterios, leyéndolos desde la perspectiva del Modelo de Procesamiento Adaptativo de Información (PAI).

\section{Criterio 1. Esfuerzos frenéticos para evitar un abandono real o imaginario}

Si en ocasiones la proximidad, el afecto y el cuidado le son negados, o recibe mensajes contradictorios al respecto, el niño buscará con desesperación al cuidador. Los niños cuyo progenitor, aunque esté presente, no siempre está emocionalmente disponible estarán preocupados por la vinculación con los demás cuando sean adultos (Ogden, Minton y Pain, 2006) y pueden ser vistos como dependientes o incluso pegajosos. Esto se manifestará sobre todo en el apego preocupado y el desorganizado, los más relacionados con la patología límite.

También podemos encontrar manifestaciones más complejas que van acompañadas de defensas que pueden expresarse de manera indirecta: el paciente que no se siente capaz de mantener una relación que por otro lado necesita desesperadamente, pudiendo alternar entre muestras de debilidad y amenazas, un individuo que en lo relacional puede ser extremadamente dependiente de nuestra aprobación, reaccionando de forma extrema o desproporcionada ante lo que entiende como falta de interés o un paciente que presenta dificultades en sus relaciones de pareja actuales. Desde la perspectiva de EMDR no vamos a ver únicamente un "paciente difícil» o un «problema de pareja», sino que detrás de eso entenderemos que existe una información disfuncional que sigue activa, por ejemplo: una madre depresiva, incapaz de sintonizar con las necesidades del niño, o una madre ansiosa, invasiva y sobreprotectora con la que no pudo desarrollarse una diferenciación entre el self y los otros.

\section{Criterio 2. Patrón de relaciones interpersonales inestables e intensas caracterizado por la alternancia entre los extremos de idealización y devaluación}

En el apego inseguro ambivalente, la estrategia conductual del niño será de este estilo: como no puedo predecir qué va a hacer mi cuidador, si me cuelgo de su cuello (con llanto, grito, pataleo) por lo menos me aseguro de que va a estar presente. En este sentido el patrón será más o menos estable u organizado. Cuando estamos trabajando con un paciente adulto, 
identificar dónde se aprendieron estas conductas, o incluso tomar conciencia de lo desadaptativo de las mismas, puede no ser sencillo. La tendencia de los individuos con TLP no es a establecer vínculos con personas con apego seguro, capaces de vincularse de modo sano. Por ello que será necesario un trabajo psicoeducativo y aprender en base a una relación terapéutica segura y consistente, cuales son los patrones adecuados para funcionar en las relaciones. A la hora de trabajar con EMDR, que esta información adaptativa esté en el sistema es esencial, o de otro modo, las redes de memoria disfuncionales no podrán ser procesadas eficazmente.

Cuando hablamos de apego desorganizado, en cambio, vemos que el niño no puede mostrar una conducta uniforme y consistente: no sería adaptativo en el entorno que le rodea. La oscilación del niño entre la activación del sistema de apego y el de defensa es complementaria a la del progenitor. Para poder apegarse a un progenitor inconsistente y agresivo, el niño no tiene a veces otra opción que idealizarlo de cara a construir una imagen parental en ocasiones muy distante de la realidad. Muchos pacientes nos hablan de padres o familias "perfectas», negando o disociando los elementos que no cuadran con esta imagen. Los aspectos atemorizantes de la figura parental son almacenados en otro estado mental, asociado ya no al apego, sino a los sistemas biológicos de defensa. Ambos estados mentales, mediados por estructuras y sistemas neurobiológicos diferentes, pueden alternar a veces sin solución de continuidad, produciendo los drásticos cambios de la idealización a la devaluación que se observan en estos pacientes tanto en sus relaciones personales como en la relación terapéutica. Para trabajar con EMDR hemos de buscar las experiencias que se relacionan con ambos polos: los mejores momentos con el progenitor abusivo (Knipe, 2008) y los recuerdos del daño más fácilmente identificables como traumáticos. En algunos casos estas situaciones se abordarán con herramientas más propias de los trastornos disociativos (González y Mosquera, 2012).
Criterio 3. Alteración de la identidad: auto-imagen o sentido de sí mismo acusada y persistentemente inestable

Para algunos pacientes el diagnóstico es una seña de identidad (Mosquera 2004, 2010), a la que se aferran en buena parte debido a la ausencia de un autoconcepto sólido y a la dificultad que encuentran en contactar realmente con sus emociones y necesidades. Con frecuencia no tienen establecida una diferenciación entre ellos mismos (self) y el mundo externo, como resultado de una relación de apego temprana disfuncional. En otros casos, la alteración de la identidad responde a síntomas disociativos graves que pueden no resultar evidentes a menos que se exploren específicamente. En algunos pacientes veremos lo que Fonagy (2000) y otros autores denominan «experiencia extraña» dentro del propio yo: ideas o sentimientos que se experimentan como parte del propio yo pero que no parecen pertenecerle. Estos fenómenos intrusivos son el elemento central de la sintomatología disociativa según Dell (2008).

Muchas intervenciones nos ayudan a trabajar esta área con EMDR. Korn y Leeds (2002) demostraron la eficacia del desarrollo e instalación de recursos, un procedimiento en el que se exploran con el paciente lo que se denominan recursos personales, las características positivas de su personalidad (algo extremadamente difícil de identificar para estos individuos), para desarrollar más las características adaptativas menos evolucionadas. Cuando la alteración de la identidad representa una auténtica fragmentación del self, es necesario trabajar con esta disociación estructural de la personalidad (Mosquera, González y Van der Hart, 2010) en la línea del trabajo con los trastornos disociativos (González y Mosquera, 2012).

\section{Criterio 4. Impulsividad en al menos dos áreas que son potencialmente dañinas para sí mimo (Ej.: gastos, sexo, abuso de sustancias, conducción temeraria, atracones de comida...)}

Un niño que es reprimido o castigado por su cuidador cuando se enfada, aprende a contener 
y esconder su rabia. Puede que incluso aprenda a no sentirla, porque mostrar rabia puede ser peligroso o hacer daño. Si existe un progenitor agresivo, la rabia se asocia a éste y se rechaza. Al rechazarse, esta emoción no puede integrarse con el resto de las funciones mentales. Esto produce en algunos casos, una alternancia entre la contención y disociación de la rabia y su expresión no modulada e incontrolada.

En este estado mental (enfado) el paciente puede tener mayor acceso a recuerdos relacionados con las situaciones de agresión vividas en la infancia (memoria dependiente de estado) que en algunos casos no son accesibles, ni siquiera parcialmente, en un estado emocional de calma (amnesia disociativa). Muchos pacientes límite no recuerdan o tienen recuerdos borrosos o fragmentados de sus conductas auto o heteroagresivas: saben que sucedió, pero no el proceso interno, lo que dijeron o hicieron. Estos fenómenos que con frecuencia son interpretados como intentos deliberados de eximirse de responsabilidad o como llamadas de atención, constituyen con frecuencia amnesias genuinas.

En EMDR se buscarían aquellas experiencias que están en la base de la conducta. Por ejemplo, para trabajar la rabia no nos centraríamos en la reacción actual, sino en los recuerdos de situaciones de agresividad verbal o física sufridos a lo largo de la vida, o en las experiencias de amenaza o humillación que despertaron una reacción de rabia que no pudo llevarse a cabo en ese momento. Sin embargo, como comentábamos en otros apartados, si esta conducta está muy disociada, es necesario trabajar antes el síntoma disociativo. Por ejemplo conectando con la parte de la personalidad que contiene la rabia, o trabajando la fobia a esa emoción por parte del paciente (de su parte aparentemente normal).

\section{Criterio 5. Comportamientos intensos \\ o amenazas suicidas recurrentes, o comportamiento de automutilación}

En un apego inseguro ambivalente o desorganizado las necesidades del niño no son fácil- mente percibidas por su cuidador. Muchas veces, el niño ha de gritar más alto o durante más tiempo para hacerse notar. Al ser esta la pauta habitual, el niño aprende que sus demandas tienen que ser llamativas e insistentes. Otras veces renuncia desde una indefensión aprendida, y llega a ignorar sus necesidades o a tener grandes dificultades para reconocerlas. En edades posteriores No sabrá cuidarse porque no pudo aprender un patrón de autocuidado adecuado. Como terapeutas EMDR nunca entenderemos estas conductas como "caprichosas e innecesarias llamadas de atención». Siempre estaremos viendo más allá, intentando entender cómo se originó ese patrón.

Las autolesiones pueden responder también a una dificultad de autorregulación y de tolerar las emociones negativas. Los pacientes con frecuencia refieren que se provocan dolor físico porque les resulta más soportable que el dolor emocional (Mosquera 2008). Por medio de las autolesiones tratan de salir de una emoción displacentera, un recuerdo traumático o una experiencia de despersonalización.

De nuevo estas conductas pueden en algunos TLP estar asociados a partes disociativas, lo que nos resultará evidente tras la exploración de la secuencia que lleva a la autolesión. El rechazo a las situaciones traumáticas vividas o la negación de las mismas puede llevar a una disociación extrema, donde partes internas reproducen la conducta del abusador, agrediendo al individuo como una reproducción del maltrato temprano. De nuevo esta situación nos llevaría a una planificación terapéutica más próxima a la que utilizamos con los trastornos disociativos.

\section{Criterio 6. Inestabilidad afectiva debida a una notable reactividad del estado de ánimo (por ej.: episodios de intensa disforia, irritabilidad o ansiedad, que suelen durar unas horas y raras veces unos días)}

La regulación emocional no es un proceso que se desarrolle sólo, sino que es adquirido 
desde las primeras etapas de la infancia a través de la reacción diádica cuidador-niño (Schore, 2003a, 2003b). La relación de apego sana está basada en un adulto capaz de sintonizar con el niño y consistente en sus actitudes, que ayuda al niño a modular sus reacciones emocionales. Un cierto grado de malestar es adaptativo, ya que por medio de él los niños aprenden a tolerar la frustración y a demorar la gratificación. Pero el cuidador ha de ayudar posteriormente al niño a recuperar el equilibrio. De este modo el niño (y el futuro adulto en el que se convertirá) aprende a mantener sus emociones dentro de lo que se ha denominado "ventana de tolerancia» que implica un nivel de activación adecuado para adaptarse a las situaciones y resolverlas de modo adecuado (Ogden, Minton y Pain 2006).

Aunque existen factores biológicos que contribuyen al desarrollo de dificultades para mantener una regulación emocional adecuada (Mosquera et al., 2011) podremos comprobar que este problema también está motivado por muchas situaciones de trauma preverbal, de falta de sintonía que no empiezan en los primeros eventos que el paciente nos puede contar, sino en aquellos que se produjeron cuando era demasiado pequeño para poder tener memoria biográfica.

También puede haber una marcada hipersensibilidad ante desencadenantes ambientales, que pueden tener en parte una base postraumática (disparadores). Gestos, actitudes, expresiones en el otro que recuerdan al paciente expresiones del cuidador en la infancia, pueden disparar reacciones que en un adulto parecen desproporcionadas y fuera de contexto. Un tono de voz elevado, por ejemplo, producía en una paciente una reacción de bloqueo intensa, y posteriormente le generaba un estado depresivo y una intensa culpa. Ella podía reconocer a cierto nivel que su reacción no era proporcionada a comentarios que su pareja o un compañero de trabajo le habían hecho, pero su comportamiento, por el contrario, encajaba perfectamente en la reacción de una niña ante un padre extremadamente autoritario y crítico como con el que creció. De niña sus gritos la hacían sentirse literalmente paralizada y el modo en que ella misma se culpabilizaba se parecía mucho a los frecuentes comentarios críticos y hostiles que escuchaba de su padre. Dado que ella nunca aprendió a manejar su rabia esto produjo una carencia de asertividad, por lo que este tipo de reacciones no evolucionaron al mismo nivel de otras funciones y habilidades, permaneciendo en cierto modo «congeladas en el tiempo» y conservando ciertas características de la reacción infantil de allí y entonces ante un padre al que no podía enfrentarse, pese a que aquí y ahora estaba ante un adulto frente al que tenía otras opciones y que no se parecía demasiado a su progenitor. El procesamiento de estos recuerdos con EMDR generará modificaciones en los patrones actuales de conducta.

\section{Criterio 7. Sentimientos crónicos de vacío}

A nivel hipotético, los sentimientos crónicos de vacío pueden corresponderse con la ausencia de sintonía con el progenitor. Un apego seguro crea en el niño un sentimiento de seguridad interna y de conexión con los otros. Cuando los pacientes con TLP describen las primeras experiencias vitales asociadas a esos sentimientos de vacío comentan momentos de soledad, de sentirse invisibles, de que nadie se daba cuenta realmente de cómo se sentían. Un progenitor desbordado por sus propios conflictos y dificultades puede no ser capaz de ver realmente las necesidades del niño, o no poder diferenciarlas de las suyas propias. Desde EMDR trabajamos los recuerdos traumáticos que generan y alimentan este síntoma.

\section{Criterio 8. Ira inapropiada e intensa o dificultades para controlar la ira (por ej.: muestras frecuentes de mal genio, enfado constante, peleas físicas recurrentes)}

Algunas de las personas con este diagnóstico tienen respuestas impredecibles, consistentes en cambios bruscos de humor o repentinas explosiones emocionales. Estas explosiones pueden ser verbales, físicas o combinadas. 
Muchos pacientes reproducen mediante estas reacciones, los modelos de expresión de la rabia disfuncionales con los que crecieron. Las oscilaciones entre actitudes de sumisión ante una pareja maltratadora y episodios de rabia incontrolada tienen a veces muchas similitudes con los roles de ambos progenitores durante su infancia: por ejemplo, mostrarse vulnerables, dependientes e indefensos como lo era su madre, ante las agresiones verbales y en ocasiones físicas del padre. Esto se complica más cuando el paciente reproduce estas conductas y posteriormente siente una intensa culpa. Siguiendo el ejemplo, el paciente por un lado evita comportarse como el padre y se siente posicionado del lado de la madre, pero el rechazo de su propia rabia (por identificarla con el padre), le impide el manejo adecuado de esta emoción, por lo que se acumula y estalla en modos desproporcionados e inadecuados.

Adquirir perspectiva de la conexión entre estas conductas y la historia del paciente puede ayudar a evitar una repetición automática y no reflexiva.

En algunos casos, esta impulsividad, como hemos señalado anteriormente, puede representar también un rasgo temperamental de base más biológica, que puede precisar un apoyo farmacológico específico.

\section{Criterio 9. Ideación paranoide transitoria relacionada con el estrés o síntomas disociativos graves}

Este es uno de los criterios más ignorado y de mayor relevancia en los casos de TLP graves. Como hemos comentado antes, muchas veces los gestos, palabras o detalles aparentemente insignificantes actúan como disparadores traumáticos o señales de alerta relacionadas con experiencias tempranas por eso es tan importante entender de dónde viene el síntoma. La reacción defensiva del paciente, la desconfianza o la aparente resistencia, incomprensible y desproporcionada desde el aquí y ahora, cobra todo su significado cuando lo entendemos desde el allí y entonces.
Entre los síntomas disociativos frecuentes destacan las alucinaciones auditivas, que corresponden a estados mentales completamente disociados, los cuales actúan en cierto modo como «conciencias paralelas». En otras ocasiones las voces reproducen comentarios de figuras de la vida del sujeto, y van más en la línea de flashbacks auditivos. Podemos también encontrar despersonalización, amnesias y diversos tipos de intrusiones. Es importante que una exploración exhaustiva de estos aspectos forme parte de la evaluación inicial en estos pacientes, donde la frecuencia de síntomas disociativos supera con mucho la de otros trastornos mentales.

\section{Conclusiones}

Integrar nuestros conocimientos sobre la traumatización temprana y grave con el modelo de procesamiento adaptativo de información en el que se fundamenta la terapia EMDR nos aporta las bases desde las que se elaborará el plan terapéutico. El elemento central de este trabajo es una conceptualización amplia de los casos y un trabajo que no está centrado en los síntomas, sino en identificar las experiencias que quedaron almacenadas disfuncionalmente y están produciendo los problemas actuales y entender cómo se llega de estas experiencias a los síntomas y conductas presentes. Se procesarán estos recuerdos pasados que están alimentando, manteniendo y activando los síntomas, pero también las situaciones presentes y los desafíos futuros.

Desde este mismo paradigma se pueden abordar todos los trastornos de personalidad, así como diversas patologías del Eje I. Aunque el diagnóstico clínico es un elemento a tener en cuenta cuando se trabaja con EMDR, y es desde luego necesario para la comprobación y comparación empírica de resultados, su importancia es menor que en abordajes específicamente dirigidos a los síntomas. Como comentábamos, aunque en el TLP la base traumática es quizás la más elevada de todos los trastornos de personalidad, en el resto de los trastornos del eje II también vemos altas tasas de problemas de apego y de trauma temprano. 


\section{Referencias}

APA (1994). Diagnostic and Statistical Manual of Mental Disorders (DSM-IV-TR). Washington, DC: Autor.

Ball, J. S. y Links, P. S. (2009). Borderline personality disorder and childhood trauma: evidence for a causal relationship. Current Psychiatry Reports, 11(1), 63-68.

Bateman, A. y Fonagy, P. (2004). Psychotherapy for borderline personality disorder: Mentalization based treatment. Oxford: Oxford University Press.

Battle, C. L., Shea, M. T., Johnson, D. M., Yen, S., Zlotnick, C., Zanarini, M. C. y Morey, L. C. (2004). Childhood maltreatment associated with adult personality disorders: findings from the Collaborative Longitudinal Personality Disorders Study. Journal of Personality Disorders. 18, 193-211

Bisson, J. y Andrew, M. (2007). Psychological treatment of post-traumatic stress disorder (PTSD). Cochrane Database of Systematic Reviews, 3. doi: 10.1002/14651858. CD003388.pub3.

Blizard, R. A. (2003). Disorganized attachment, development of dissociated self states and a relational approach to treatment. Journal of Trauma and Dissociation, 4(3), 27-50.

Bradley, R., Greene, J., Russ, E., Dutra, L. y Westen, D. (2005). A multidimensional meta-analysis of psychotherapy for PTSD. American Journal of Psychiatry, 162, 214-227.

Davidson, P. R. y Parker, K. C. H. (2001). Eye movement desensitization and reprocessing (EMDR): A metaanalysis. Journal of Consulting and Clinical Psychology, 69, 305-316.

Driessen M., Beblo T., Reddemann L., Rau H., Lange W., Silva A. y Ratzka S. (2002). Is the borderline personality disorder a complex posttraumatic stress disorder? - The state of research. Nervenarzt, 73(9), 820-829.

Foa, E. B., Keane, T. M. y Friedman M. J. (2000). Effective treatments for PTSD: Practice guidelines from the International Society for Traumatic Stress Studies. New York: Guilford Press. Fonagy, P. y Bateman, A. (2007). Teoría del apego y modelo orientado a la mentalización del trastorno límite de la personalidad. En Tratado de los Trastornos de la Personalidad (pp. 189-203) [Textbook of personality disorders]. Barcelona, España: Masson.
Fonagy, P. (2000). Attachment and borderline personality disorder. Journal of the American Psychoanalytic Association, 48, 1129-1146; discussion 1175-1187.

Fossati, A., Madeddu, F. y Maffei, C. (1999). Borderline personality disorder and childhood sexual abuse: A meta-analytical study. Journal of Personality Disorder, 13, 268-280.

González, A. y Mosquera, D. (2012). EMDR y Disociación: El abordaje progresivo [EMDR and Dissociation: The progressive approach]. Madrid, España: Pléyades.

Goodman, M. y Yehuda, R. (2002). The relationship between psychological trauma and borderline personality disorder. Psychiatric Annals, 33, 337-345.

Grover K. E., Carpenter L. L., Price L. H., Gagne G. G., Mello A. F., Mello M. F. y Tyrka A.R. (2007). The relationship between childhood abuse and adult personality disorder symptoms. Journal of Personality Disorder, 21(4), 442-447.

Gunderson, J. (1984). Borderline personality disorder. Washington, DC: American Psychiatric Association.

Gunderson, J. (1996). The borderline patient's intolerance of aloneness: Insecure attachments and therapist availability. American Journal of Psychiatry, 153, 752-758.

Guttman, H. y Laporte, L. (2002). Family members'retrospective perceptions of intrafamiliar retrospectives. Contemporany Family Therapy: An International Journal, 24(3), 505-521.

Harned M. S., Rizvi S. L. y Linehan M. M. (2010). Impact of co-occurring posttraumatic stress disorder on suicidal women with borderline personality disorder. American Journal of Psychiatry, 167(10), 1210-1217.

Hill, A. B. (1965). The environment and disease: association or causation? Proceedings of the Royal Society of Medicine 58, 295-300.

Johnson, J. G., Cohen, P., Brown, J., Smailes, E. M. y Bernstein, D. P. (1999). Childhood maltreatment increases risk for personality disorders during early adulthood. Archives of General Psychiatry, 56, 600-606.

Kingdon, D. G., Ashcroft, K., Bhandari, B., Gleeson, S., Warikoo, N., Symons y Mehta, R. (2010). 
Schizophrenia and borderline personality disorder: similarities and differences in the experience of auditory hallucinations, paranoia, and childhood trauma. Journal of Nervous y Mental Disease, 198(6), 399-403.

Korn, D. L. y Leeds, A. M. (2002). Preliminary Evidence of Efficacy for EMDR Resource Development and Installation in the Stabilization Phase of Complex Posttraumatic Stress Disorder. Journal of Clinical Psychology, 58(12), 1465-1487.

Knipe, J. (2008). EMDR toolbox. Taller preconferencia del IX EMDR Europe Conference. Londres.

Laporte, L. y Guttman, H. (1996). Traumatic childhood experiences as risk factors for borderline and other personality disorders. Journal of Personality Disorders, 10, 247-259.

Liotti, G. (2004). Trauma, dissociation and disorganized attachment: three strands of a single braid. Psychotherapy Theory, Research and Practice Training, 41, 472-486.

Lyons-Ruth. K., Yellin, C., Melnick, S. y Atwood, G. (2005). Expanding the concept of unresolved mental states: hostile/helpless states of mind on the Adult Attachment Interview are associated with disrupted mother-infant communication and infant disorganization. Deviant Psychopathology, 17, 1-23.

McLean, L. M. y R. Gallop (2003). Implications of childhood sexual abuse for adult borderline personality disorder and complex posttraumatic stress disorder. American Journal of Psychiatry, 160, 369-371.

Mosquera, D. (2004). Diamantes en Bruto I. Un acercamiento al trastorno límite de la personalidad. Manual informativo para profesionales, pacientes $y$ familiares [Rough Diamonds. Part 1. An approach to Borderline Personality Disorder. A guide for therapists, patients and families]. Madrid, España: Pléyades.

Mosquera, D. (2008). La autolesión: el lenguaje del dolor [Self-Harm: the language of pain]. Madrid, España: Pléyades.

Mosquera, D. (2010). Trastorno Límite de la Personalidad. Una aproximación conceptual a los criterios del DSM-IV- TR [A conceptual approach to the DSM-IV-TR diagnostic criteria]. Revista Persona, 10(2), 7-22.
Mosquera, D. y González A. (2011). Del apego temprano al TLP [From early attachment to BPD]. Revista Mente y Cerebro, 18-27.

Mosquera, D., González A. y Van der Hart, O. (2011). Borderline Personality Disorder, childhood trauma and structural dissociation of the personality. Rev. Persona, FUNDAP, 44-73.

Nickell, A. D., Waudby, C. J. y Trull, T. J. (2002). Attachment, parental bonding, and Borderline Personality Disorder features in young adults. Journal of Personality Disorders, 16(2), 148-159.

Ogden, P., Minton, K. y Pain, C. (2006). Trauma and the body: A sensorimotor approach to psychotherapy. New York: Norton.

Rogosch, F. A. y Cicchetti, D. (2005). Child maltreatment, attention networks and potential precursors to borderline personality disorder. Deviant Psychopathology, 17, 1071-1089.

Sabo, A. N. (1997). Etiological significance of associations between childhood trauma and borderline personality disorder: Conceptual and clinical implications. Journal of Personality Disorders, 11, 50-70.

Sansone, R. A., Sansone, L. A. y Wiederman, M.W. (1995). Trauma, borderline personality, and selfharm behaviors. Archives of Family Medicine, 4(12), 1000-1002.

Schore, A. (2001). The effects of early relational trauma on right brain development, affect regulation and infant mental health. Infant Mental Health, 22, 201-269.

Schore, A. (2003a). Affect dysregulation and disorders of the self. New York: Norton.

Schore, A. (2003b). Affect regulation and the repair of the self. New York: Norton.

Seidler, G. H. y Wagner, F. E. (2006). Comparing the efficacy of EMDR and trauma-focused cognitive-behavioral therapy in the treatment of PTSD: a meta-analytic study. Psychological Medicine, 36, 1515-1522.

Shapiro, F. (2001). Eye movement desensitization and reprocessing: Basic principles, protocols, and procedures ( $2^{\mathrm{a}} \mathrm{Ed}$.). New York: Guilford Press.

Shapiro, F. (2007). EMDR and case conceptualization from an adaptive information processing perspective. En F. Shapiro, F. Kaslow y L. Maxfield (Eds.), Handbook of EMDR and family therapy processes (pp. 3-36). New York: Wiley. 
Siegel, D. J. (2002). The developing mind and the resolution of trauma: Some ideas about information processing and an interpersonal neurobiology of psychotherapy. En F. Shapiro (Ed.), EMDR as an integrative psychotherapy approach: Experts of diverse orientations explore the paradigm prism (pp. 85-122). Washington, DC: American Psychological Association Press.

Silk, K. R., Lee, S., Hill, E. M. D. y Lohr, N. E. (1995). Borderline personality disorder symptoms and severity of sexual abuse. American Journal of Psychiatry, 152, 1059-1064.

Spitzer, C., Effler, K. y Freyberger, H. J. (2000). Posttraumatic stress disorder, dissociation and self-destructive behavior in borderline patients. Zeitschrift für Psychosomatische Medizin und Psychotherapie 46(3), 273-285.

Stickgold, R. (2002). EMDR: A putative neurobiological mechanism of action. Journal of Clinical Psychology, 58, 61-75.

Tyrka, A. R., Wyche, M. C., Kelly, M. M., Price, L. H. y Carpenter, L. L. (2009). Childhood maltreatment and adult personality disorder symptoms: influence of maltreatment type. Psychiatry Research, 65(3), 281-287.

Van der Kolk, B. A. (2002). Beyond the talking cure: Somatic experience and subcortical imprints in the treatment of trauma. En F. Shapiro (Ed.), EMDR as an integrative psychotherapy approach:
Experts of diverse orientations explore the paradigm prism (pp. 57-84). Washington, DC: American Psychological Association Press.

Van Etten, M. y Taylor, S. (1998). Comparative efficacy of treatments for posttraumatic stress disorder: A metaanalysis. Clinical Psychology and Psychotherapy, 5, 126-144.

Yen, S., Shea, M. T., Battle, C. L., Johnson, D. M., Zlotnick, C., Dolan-Sewell, R. y McGlashan, T. H. (2002). Traumatic exposure and posttraumatic stress disorder in borderline, schizotypal, avoidant, and obsessive-compulsive personality disorders: findings from the collaborative longitudinal personality disorders study. Journal of Nervous y Mental Disease 190, 510-518.

Zanarini, M. C. (2000). Childhood experiences associated with the development of borderline personality disorder. Psychiatric Clinics of North America, 23, 89-101.

Zanarini, M.C., Ruser, T. F. Frankenburg, F. R., Hennen, J. y Gunderson, J. G. (2000). Risk factors associated with the dissociative experiences of borderline patients. Journal of Nervous y Mental Disease, 188(1), 26-30.

Zweig-Frank, H. y Paris, J. (1991). Parents' emotional neglect and overprotection according to the recollections of patients with borderline personality disorder. American Journal of Psychiatry, 148, 648-651. 
